Supporting Information

\title{
Insights into the Photoelectron Spectroscopy of Chlorofluoroethenes Studied by Density-Functional and Coupled-Cluster Theories
}

Cyong-Huei Huang, Shang-Yi Chou, Shiu-Bau Jang, Yu-Chieh Lin, Chien-En Li, Chiing-Chang Chen, and Jia-Lin Chang*

Department of Science Education and Application, National Taichung University of Education, Taichung 40306, Taiwan, Republic of China

The equilibrium structures and harmonic vibrational frequencies computed by the B3PW91 and TD-B3PW91 methods with the aug-cc-pVTZ basis set, and those obtained by the CCSD and EOM-CCSD methods with the cc-pVDZ or cc-pVTZ basis set are given in Tables S1-S6. Tables S7-S9 list the vibrational structures of the photoelectron spectra corresponding to the ground state of $\mathrm{C}_{2} \mathrm{H}_{2} \mathrm{FCl}^{+}$. The computed CCSD(T) energies are given in Table S10. Figures S1-S5 depict the simulated photoelectron spectra mentioned but not shown in the text.

Except for trans $-\mathrm{C}_{2} \mathrm{H}_{2} \mathrm{FCl}^{+}\left(\tilde{A}^{2} \mathrm{~A}^{\prime}\right)$, the simulated photoelectron spectra of the first excited state of $\mathrm{C}_{2} \mathrm{H}_{2} \mathrm{FCl}^{+}$with the EOM-CCSD method are not in harmony with the experiments (Figs. S3-S5). This implies that higher-level of basis set is required. However, it will be very time-consuming and whether the simulated spectra can be 
improved need to be further investigated.

TABLE S1. Equilibrium Geometries of $1,1-\mathrm{C}_{2} \mathrm{H}_{2} \mathrm{FCl}$ and Its Cations

\begin{tabular}{lllllll}
\hline State $^{a}$ & $\mathrm{X}$ & $\mathrm{X}$ & $\mathrm{X}+$ & $\mathrm{A}+$ & $\mathrm{A}+$ & $\mathrm{A}+$ \\
\hline Parameter $^{b}$ & $\mathrm{~B} 3 \mathrm{PW} 91$ & $\mathrm{CCSD}^{c}$ & $\mathrm{~B} 3 \mathrm{PW} 9$ & $\mathrm{~B} 3 \mathrm{PW} 9$ & $\mathrm{TD}-\mathrm{B} 3 \mathrm{PW} 1$ & EOM-CCSD $^{c}$ \\
\hline $\mathrm{R}_{\mathrm{CC}}$ & 131.81 & 132.19 & 140.34 & 129.93 & 131.09 & 130.34 \\
$\mathrm{R}_{\mathrm{CH} 1}$ & 108.04 & 107.82 & 108.50 & 109.06 & 108.61 & 108.76 \\
$\mathrm{R}_{\mathrm{CH} 2}$ & 107.76 & 107.57 & 108.24 & 108.51 & 108.15 & 108.27 \\
$\mathrm{R}_{\mathrm{CF}}$ & 132.88 & 132.64 & 127.80 & 128.57 & 128.72 & 129.43 \\
$\mathrm{R}_{\mathrm{CCl}}$ & 171.89 & 172.05 & 164.58 & 184.89 & 181.99 & 179.38 \\
$\mathrm{~A}_{\mathrm{CCH} 1}$ & $119.5^{\circ}$ & $119.4^{\circ}$ & $119.0^{\circ}$ & $118.3^{\circ}$ & $117.9^{\circ}$ & $118.6^{\circ}$ \\
$\mathrm{A}_{\mathrm{CCH} 2}$ & $120.5^{\circ}$ & $120.3^{\circ}$ & $120.0^{\circ}$ & $123.3^{\circ}$ & $122.6^{\circ}$ & $122.6^{\circ}$ \\
$\mathrm{A}_{\mathrm{CCF}}$ & $122.8^{\circ}$ & $122.7^{\circ}$ & $119.2^{\circ}$ & $135.8^{\circ}$ & $131.7^{\circ}$ & $135.6^{\circ}$ \\
$\mathrm{A}_{\mathrm{CCCl}}$ & $125.6^{\circ}$ & $125.4^{\circ}$ & $123.6^{\circ}$ & $117.8^{\circ}$ & $123.2^{\circ}$ & $116.2^{\circ}$ \\
\hline
\end{tabular}

${ }^{a} \mathrm{X}$ stands for the neutral ground state, $\mathrm{X}+$ for the cationic ground state, $\mathrm{A}+$ for the first excited state of cation.

${ }^{b} \mathrm{R}$ stands for bond lengths (in pm) and A for bond angles.

${ }^{c}$ The basis set used is cc-pVTZ.

TABLE S2. Equilibrium Geometries of $C i s-\mathrm{C}_{2} \mathrm{H}_{2} \mathrm{FCl}$ and Its Cations

\begin{tabular}{lllllll}
\hline State $^{a}$ & $\mathrm{X}$ & $\mathrm{X}$ & $\mathrm{X}+$ & $\mathrm{A}+$ & $\mathrm{A}+$ & $\mathrm{A}+$ \\
\hline Parameter $^{b}$ & $\mathrm{~B} 3 \mathrm{PW} 91$ & $\mathrm{CCSD}^{c}$ & $\mathrm{~B} 3 \mathrm{PW} 91$ & $\mathrm{~B} 3 \mathrm{PW} 91$ & $\mathrm{TD}-\mathrm{B} 3 \mathrm{PW} 91$ & EOM-CCSD $^{c}$ \\
\hline $\mathrm{R}_{\mathrm{CC}}$ & 132.18 & 133.94 & 139.93 & 133.05 & 133.10 & 134.35 \\
$\mathrm{R}_{\mathrm{CH} 1}$ & 108.23 & 109.42 & 108.82 & 108.59 & 108.49 & 109.68 \\
$\mathrm{R}_{\mathrm{CH} 2}$ & 107.90 & 109.21 & 108.52 & 109.70 & 108.95 & 110.01 \\
$\mathrm{R}_{\mathrm{CF}}$ & 133.10 & 133.83 & 127.30 & 130.16 & 130.05 & 131.44 \\
$\mathrm{R}_{\mathrm{CCl}}$ & 171.82 & 173.57 & 164.07 & 171.55 & 173.81 & 175.36 \\
$\mathrm{~A}_{\mathrm{CCH} 1}$ & $123.0^{\circ}$ & $122.5^{\circ}$ & $123.0^{\circ}$ & $120.7^{\circ}$ & $121.5^{\circ}$ & $121.2^{\circ}$ \\
$\mathrm{A}_{\mathrm{CCH} 2}$ & $120.4^{\circ}$ & $120.5^{\circ}$ & $119.4^{\circ}$ & $124.7^{\circ}$ & $125.5^{\circ}$ & $128.8^{\circ}$ \\
$\mathrm{A}_{\mathrm{CCF}}$ & $123.3^{\circ}$ & $123.4^{\circ}$ & $120.8^{\circ}$ & $122.7^{\circ}$ & $122.1^{\circ}$ & $121.8^{\circ}$ \\
$\mathrm{A}_{\mathrm{CCC} 1}$ & $123.9^{\circ}$ & $123.1^{\circ}$ & $122.1^{\circ}$ & $124.7^{\circ}$ & $123.3^{\circ}$ & $118.6^{\circ}$ \\
\hline
\end{tabular}

${ }^{a} \mathrm{X}$ stands for the neutral ground state, $\mathrm{X}+$ for the cationic ground state, $\mathrm{A}+$ for the first excited state of cation.

${ }^{b} \mathrm{R}$ stands for bond lengths (in pm) and A for bond angles.

${ }^{c}$ The basis set used is cc-pVDZ. 
TABLE S3. Equilibrium Geometries of Trans $-\mathrm{C}_{2} \mathrm{H}_{2} \mathrm{FCl}$ and Its Cations

\begin{tabular}{lllllll}
\hline State $^{a}$ & $\mathrm{X}$ & $\mathrm{X}$ & $\mathrm{X}+$ & $\mathrm{A}+$ & $\mathrm{A}+$ & $\mathrm{A}+$ \\
\hline Parameter $^{b}$ & B3PW91 & $\mathrm{CCSD}^{c}$ & B3PW91 & B3PW91 & TD-B3PW91 & EOM-CCSD $^{c}$ \\
\hline $\mathrm{R}_{\mathrm{CC}}$ & 132.07 & 133.93 & 139.59 & 133.38 & 133.35 & 134.94 \\
$\mathrm{R}_{\mathrm{CH} 1}$ & 108.16 & 109.38 & 108.82 & 108.87 & 108.42 & 109.70 \\
$\mathrm{R}_{\mathrm{CH} 2}$ & 108.00 & 109.28 & 108.65 & 110.75 & 109.54 & 110.78 \\
$\mathrm{R}_{\mathrm{CF}}$ & 133.86 & 134.44 & 127.48 & 129.56 & 129.73 & 130.54 \\
$\mathrm{R}_{\mathrm{CCl}}$ & 172.33 & 173.97 & 164.28 & 169.02 & 172.53 & 173.12 \\
$\mathrm{~A}_{\mathrm{CCH} 1}$ & $125.8^{\circ}$ & $125.1^{\circ}$ & $125.0^{\circ}$ & $124.1^{\circ}$ & $126.0^{\circ}$ & $125.0^{\circ}$ \\
$\mathrm{A}_{\mathrm{CCH} 2}$ & $123.0^{\circ}$ & $122.6^{\circ}$ & $121.3^{\circ}$ & $122.4^{\circ}$ & $124.9^{\circ}$ & $126.2^{\circ}$ \\
$\mathrm{A}_{\mathrm{CCF}}$ & $120.5^{\circ}$ & $120.7^{\circ}$ & $118.6^{\circ}$ & $120.5^{\circ}$ & $118.7^{\circ}$ & $120.0^{\circ}$ \\
$\mathrm{A}_{\mathrm{CCC} 1}$ & $121.3^{\circ}$ & $121.1^{\circ}$ & $119.8^{\circ}$ & $128.6^{\circ}$ & $125.5^{\circ}$ & $123.5^{\circ}$ \\
\hline
\end{tabular}

${ }^{a} \mathrm{X}$ stands for the neutral ground state, $\mathrm{X}+$ for the cationic ground state, $\mathrm{A}+$ for the first excited state of cation.

${ }^{b} \mathrm{R}$ stands for bond lengths (in pm) and A for bond angles.

${ }^{c}$ The basis set used is cc-pVDZ. 
TABLE S4. Harmonic Vibrational Frequencies (in $\mathrm{cm}^{-1}$ ) of $1,1-\mathrm{C}_{2} \mathrm{H}_{2} \mathrm{FCl}$ and Its

\begin{tabular}{clccccc}
\multicolumn{7}{c}{ Cations } \\
State $^{a}$ & X & X & X+ & A+ & A+ & A+ \\
\hline Mode & B3PW91 & CCSD $^{b}$ & B3PW91 & B3PW91 & TD-B3PW91 & EOM-CCSD $^{b}$ \\
\hline$v_{1}$ & 3291 & 3321 & 3275 & 3196 & 3256 & 3241 \\
$v_{2}$ & 3187 & 3215 & 3149 & 3080 & 3143 & 3127 \\
$v_{3}$ & 1718 & 1748 & 1503 & 1791 & 1812 & 1801 \\
$v_{4}$ & 1398 & 1431 & 1421 & 1349 & 1394 & 1402 \\
$v_{5}$ & 1194 & 1244 & 1310 & 1094 & 1173 & 1133 \\
$v_{6}$ & 959 & 983 & 998 & 906 & 947 & 935 \\
$v_{7}$ & 701 & 715 & 759 & 488 & 593 & 603 \\
$v_{8}$ & 436 & 440 & 460 & 341 & 374 & 389 \\
$v_{9}$ & 372 & 375 & 366 & 173 & 248 & 215 \\
$v_{10}$ & 860 & 884 & 936 & 881 & 932 & 959 \\
$v_{11}$ & 721 & 740 & $\underline{361^{c}}$ & 740 & 712 & 786 \\
$v_{12}$ & 541 & 538 & $\underline{567}$ & 479 & 500 & 474 \\
\hline
\end{tabular}

${ }^{a} \mathrm{X}$ stands for the neutral ground state, $\mathrm{X}+$ for the cationic ground state, $\mathrm{A}+$ for the first excited state of cation.

${ }^{b}$ The basis set used is cc-pVTZ.

${ }^{c}$ The underlines denote normal modes whose orders are changed in comparison to those of the ground state. 
TABLE S5. Harmonic Vibrational Frequencies (in $\mathrm{cm}^{-1}$ ) of $C i s-\mathrm{C}_{2} \mathrm{H}_{2} \mathrm{FCl}$ and Its

\begin{tabular}{|c|c|c|c|c|c|c|}
\hline \multicolumn{7}{|c|}{ Cations } \\
\hline State $^{a}$ & $\mathrm{X}$ & $X$ & $\mathrm{X}+$ & $\mathrm{A}+$ & $\mathrm{A}+$ & $\mathrm{A}+$ \\
\hline Mode & B3PW91 & $\operatorname{CCSD}^{b}$ & B3PW91 & B3PW91 & TD-B3PW91 & EOM-CCSD \\
\hline$v_{1}$ & 3239 & 3277 & 3194 & $\underline{3015^{c}}$ & $\underline{3115}$ & $\underline{3184}$ \\
\hline$v_{2}$ & 3205 & 3250 & 3174 & $\underline{3187}$ & $\underline{3205}$ & $\underline{3259}$ \\
\hline$v_{3}$ & 1719 & 1757 & 1570 & 1625 & 1697 & 1686 \\
\hline$v_{4}$ & 1351 & 1375 & 1396 & 1295 & 1325 & 1311 \\
\hline$v_{5}$ & 1256 & 1270 & 1320 & 1240 & 1265 & 1251 \\
\hline$v_{6}$ & 1082 & 1093 & 1129 & 1027 & 1075 & 1066 \\
\hline$v_{7}$ & 815 & 825 & 896 & 639 & 696 & 725 \\
\hline$v_{8}$ & 664 & 665 & 687 & 607 & 615 & 626 \\
\hline$v_{9}$ & 195 & 197 & 211 & 157 & 159 & 143 \\
\hline$v_{10}$ & 896 & 893 & 932 & 914 & 929 & 959 \\
\hline$v_{11}$ & 753 & 766 & 758 & 667 & 687 & 661 \\
\hline$v_{12}$ & 461 & 453 & 317 & 419 & 435 & 421 \\
\hline
\end{tabular}

${ }^{a} \mathrm{X}$ stands for the neutral ground state, $\mathrm{X}+$ for the cationic ground state, $\mathrm{A}+$ for the first excited state of cation.

${ }^{b}$ The basis set used is cc-pVDZ.

${ }^{c}$ The underlines denote normal modes whose orders are changed in comparison to those of the ground state. 
TABLE S6. Harmonic Vibrational Frequencies (in $\mathrm{cm}^{-1}$ ) of Trans $-\mathrm{C}_{2} \mathrm{H}_{2} \mathrm{FCl}$ and Its

\begin{tabular}{|c|c|c|c|c|c|c|}
\hline \multicolumn{7}{|c|}{ Cations } \\
\hline State $^{a}$ & $\mathrm{X}$ & $X$ & $\mathrm{X}+$ & $\mathrm{A}+$ & $\mathrm{A}+$ & $\mathrm{A}+$ \\
\hline Mode & B3PW91 & $\mathrm{CCSD}^{b}$ & B3PW91 & B3PW91 & TD-B3PW91 & EOM-CCSD \\
\hline$v_{1}$ & 3226 & 3267 & 3180 & $\underline{2900}$ & $\underline{3041}$ & $\underline{3083}$ \\
\hline$v_{2}$ & 3218 & 3259 & 3176 & $\underline{3135}$ & $\underline{3203}$ & $\underline{3245}$ \\
\hline$v_{3}$ & 1708 & 1743 & 1573 & 1618 & 1676 & 1665 \\
\hline$v_{4}$ & 1318 & 1327 & 1255 & 1306 & 1335 & 1338 \\
\hline$v_{5}$ & 1236 & 1239 & 1291 & $\underline{1036}$ & $\underline{1139}$ & $\underline{1108}$ \\
\hline$v_{6}$ & 1151 & 1173 & 1310 & $\underline{1254}$ & $\underline{1270}$ & $\underline{1264}$ \\
\hline$v_{7}$ & 886 & 896 & 991 & 739 & 731 & 765 \\
\hline$v_{8}$ & 453 & 453 & 467 & 434 & 435 & 439 \\
\hline$v_{9}$ & 269 & 272 & 277 & 258 & 253 & 242 \\
\hline$v_{10}$ & 923 & 934 & $\underline{828^{c}}$ & 954 & 967 & 995 \\
\hline$v_{11}$ & 822 & 803 & $\underline{927}$ & 688 & 721 & 670 \\
\hline$v_{12}$ & 273 & 276 & 208 & 256 & 263 & 253 \\
\hline
\end{tabular}

${ }^{a} \mathrm{X}$ stands for the neutral ground state, $\mathrm{X}+$ for the cationic ground state, $\mathrm{A}+$ for the first excited state of cation.

${ }^{b}$ The basis set used is cc-pVDZ.

${ }^{c}$ The underlines denote normal modes whose orders are changed in comparison to those of the ground state. 
TABLE S7. Vibrational Structure ${ }^{a}$ of the Photoelectron Spectrum of

\section{$1,1-\mathrm{C}_{2} \mathrm{H}_{2} \mathrm{FCl}^{+}\left(\tilde{X}^{2} A^{\prime \prime}\right)$}

\begin{tabular}{|c|c|c|c|c|c|c|c|}
\hline Band & $\Delta \mathrm{E}$ & FCF & Transition & Band & $\Delta \mathrm{E}$ & FCF & Transition \\
\hline $0^{0}$ & 0 & 0.14984 & $0^{0}$ & $\mathrm{~F}$ & 3540 & 0.01252 & $3^{1} 5^{1} 7^{1}$ \\
\hline $\mathbf{A}$ & 367 & 0.00237 & $9^{1}$ & & 3578 & 0.00546 & $4^{2} 7^{1}$ \\
\hline B & 734 & 0.00644 & $11^{2}$ & & 3661 & 0.01414 & $3^{1} 4^{1} 7^{1}$ \\
\hline B & 747 & 0.03405 & $7^{1}$ & & 3743 & 0.00954 & $3^{2} 7^{1}$ \\
\hline \multirow[t]{3}{*}{$\mathrm{C}$} & 1294 & 0.06347 & $5^{1}$ & $\mathrm{G}$ & 3883 & 0.00247 & $5^{3}$ \\
\hline & 1415 & 0.07712 & $4^{1}$ & & 4004 & 0.00504 & $4^{1} 5^{2}$ \\
\hline & 1495 & 0.00518 & $7^{2}$ & & 4087 & 0.00711 & $3^{1} 5^{2}$ \\
\hline C & 1498 & 0.10321 & $3^{1}$ & & 4125 & 0.00588 & $4^{2} 5^{1}$ \\
\hline \multirow[t]{5}{*}{$\mathrm{D}$} & 2028 & 0.00273 & $5^{1} 11^{2}$ & & 4208 & 0.01424 & $3^{1} 4^{1} 5^{1}$ \\
\hline & 2042 & 0.01869 & $5^{1} 7^{1}$ & & 4246 & 0.00404 & $4^{3}$ \\
\hline & 2149 & 0.00332 & $4^{1} 11^{2}$ & & 4287 & 0.00252 & $3^{1} 5^{1} 7^{2}$ \\
\hline & 2163 & 0.01879 & $4^{1} 7^{1}$ & & 4290 & 0.00909 & $3^{2} 5^{1}$ \\
\hline & 2232 & 0.00444 & $3^{1} 11^{2}$ & & 4328 & 0.01324 & $3^{1} 4^{2}$ \\
\hline D & 2245 & 0.02683 & $3^{1} 7^{1}$ & & 4408 & 0.00249 & $3^{1} 4^{1} 7^{2}$ \\
\hline \multirow[t]{6}{*}{ E } & 2589 & 0.01473 & $5^{2}$ & & 4411 & 0.01488 & $3^{2} 4^{1}$ \\
\hline & 2710 & 0.02666 & $4^{1} 5^{1}$ & & 4494 & 0.00579 & $3^{3}$ \\
\hline & 2789 & 0.00337 & $5^{1} 7^{2}$ & $\mathrm{H}$ & 4834 & 0.00304 & $3^{1} 5^{2} 7^{1}$ \\
\hline & 2792 & 0.03659 & $3^{1} 5^{1}$ & & 5038 & 0.00367 & $3^{2} 5^{1} 7^{1}$ \\
\hline & 2830 & 0.02104 & $4^{2}$ & & 5076 & 0.00393 & $3^{1} 4^{2} 7^{1}$ \\
\hline & 2910 & 0.00300 & $4^{1} 7^{2}$ & & 5159 & 0.00477 & $3^{2} 4^{1} 7^{1}$ \\
\hline \multirow[t]{3}{*}{$\mathbf{E}$} & 2913 & 0.05073 & $3^{1} 4^{1}$ & I & 5623 & 0.00290 & $3^{1} 4^{2} 5^{1}$ \\
\hline & 2993 & 0.00448 & $3^{1} 7^{2}$ & & 5706 & 0.00321 & $3^{2} 4^{1} 5^{1}$ \\
\hline & 2996 & 0.03190 & $3^{2}$ & & 5744 & 0.00243 & $3^{1} 4^{3}$ \\
\hline \multirow[t]{2}{*}{$\mathrm{F}$} & 3336 & 0.00535 & $5^{2} 7^{1}$ & & 5827 & 0.00369 & $3^{2} 4^{2}$ \\
\hline & 3457 & 0.00861 & $4^{1} 5^{1} 7^{1}$ & & 5909 & 0.00254 & $3^{3} 4^{1}$ \\
\hline
\end{tabular}

${ }^{a}$ Assigned according to the Franck-Condon factor computation at the B3LYP/aug-cc-pVTZ level. $\triangle \mathrm{E}$ is the excitation energy $\left(\mathrm{cm}^{-1}\right)$ relative to the adiabatic ionization energy. Bands A to I are given in Fig. 2(a). 
TABLE S8. Vibrational Structure ${ }^{a}$ of the Photoelectron Spectrum of Cis $-\mathrm{C}_{2} \mathrm{H}_{2} \mathrm{FCl}^{+}\left(\tilde{X}^{2} A^{\prime \prime}\right)$

\begin{tabular}{|c|c|c|c|c|c|c|c|}
\hline Band & $\Delta \mathrm{E}$ & FCF & Transition & Band & $\Delta \mathrm{E}$ & FCF & Transition \\
\hline $0^{0}$ & 0 & 0.14203 & $0^{0}$ & $\mathbf{F}$ & 2869 & 0.03472 & $3^{1} 5^{1}$ \\
\hline \multirow[t]{2}{*}{ A } & 213 & 0.02913 & $9^{1}$ & & 2891 & 0.00351 & $3^{1} 6^{1} 9^{1}$ \\
\hline & 426 & 0.00424 & $9^{2}$ & & 2947 & 0.01480 & $3^{1} 4^{1}$ \\
\hline \multirow[t]{2}{*}{ B } & 633 & 0.00277 & $12^{2}$ & G & 3082 & 0.00616 & $3^{1} 5^{1} 9^{1}$ \\
\hline & 683 & 0.00314 & $8^{1}$ & G & 3107 & 0.06642 & $3^{2}$ \\
\hline B & 882 & 0.02477 & $7^{1}$ & & 3160 & 0.00341 & $3^{1} 4^{1} 9^{1}$ \\
\hline $\mathrm{C}$ & 1095 & 0.00451 & $7^{1} 9^{1}$ & & 3317 & 0.00439 & $3^{1} 7^{2}$ \\
\hline $\mathrm{C}$ & 1125 & 0.02201 & $6^{1}$ & & 3320 & 0.01360 & $3^{2} 9^{1}$ \\
\hline \multirow[t]{4}{*}{ D } & 1316 & 0.03366 & $5^{1}$ & $\mathrm{H}$ & 3560 & 0.00477 & $3^{1} 6^{1} 7^{1}$ \\
\hline & 1337 & 0.00473 & $6^{1} 9^{1}$ & & 3751 & 0.00818 & $3^{1} 5^{1} 7^{1}$ \\
\hline & 1393 & 0.01874 & $4^{1}$ & & 3829 & 0.00437 & $3^{1} 4^{1} 7^{1}$ \\
\hline & 1529 & 0.00595 & $5^{1} 9^{1}$ & H & 3989 & 0.01622 & $3^{2} 7^{1}$ \\
\hline \multirow[t]{6}{*}{ D } & 1554 & 0.13921 & $3^{1}$ & & 3994 & 0.00313 & $3^{1} 5^{1} 6^{1}$ \\
\hline & 1606 & 0.00427 & $4^{1} 9^{1}$ & I & 4185 & 0.00523 & $3^{1} 5^{2}$ \\
\hline & 1764 & 0.00342 & $7^{2}$ & & 4202 & 0.00300 & $3^{2} 7^{1} 9^{1}$ \\
\hline & 1767 & 0.02853 & $3^{1} 9^{1}$ & & 4232 & 0.00549 & $3^{2} 6^{1}$ \\
\hline & 1979 & 0.00415 & $3^{1} 9^{2}$ & & 4263 & 0.00304 & $3^{1} 4^{1} 5^{1}$ \\
\hline & 2006 & 0.00524 & $6^{1} 7^{1}$ & H & 4423 & 0.01743 & $3^{2} 5^{1}$ \\
\hline \multirow[t]{3}{*}{$\mathrm{E}$} & 2198 & 0.00680 & $5^{1} 7^{1}$ & & 4501 & 0.00550 & $3^{2} 4^{1}$ \\
\hline & 2237 & 0.00322 & $3^{1} 8^{1}$ & $\mathrm{~J}$ & 4636 & 0.00310 & $3^{2} 5^{1} 9^{1}$ \\
\hline & 2275 & 0.00455 & $4^{1} 7^{1}$ & $\mathbf{J}$ & 4661 & 0.02054 & $3^{3}$ \\
\hline \multirow[t]{2}{*}{$\mathbf{E}$} & 2436 & 0.02887 & $3^{1} 7^{1}$ & $\mathbf{K}$ & 4874 & 0.00420 & $3^{3} 9^{1}$ \\
\hline & 2440 & 0.00413 & $5^{1} 6^{1}$ & $\mathbf{L}$ & 5305 & 0.00476 & $3^{2} 5^{1} 7^{1}$ \\
\hline \multirow[t]{4}{*}{$\mathrm{F}$} & 2631 & 0.00487 & $5^{2}$ & $\mathbf{M}$ & 5543 & 0.00586 & $3^{3} 7^{1}$ \\
\hline & 2648 & 0.00530 & $3^{1} 7^{1} 9^{1}$ & $\mathbf{N}$ & 5977 & 0.00568 & $3^{3} 5^{1}$ \\
\hline & 2678 & 0.01621 & $3^{1} 6^{1}$ & O & 6215 & 0.00463 & $3^{4}$ \\
\hline & 2709 & 0.00370 & $4^{1} 5^{1}$ & & & & \\
\hline
\end{tabular}

${ }^{a}$ Assigned according to the Franck-Condon factor computation at the B3LYP/aug-cc-pVTZ level. $\Delta \mathrm{E}$ is the excitation energy $\left(\mathrm{cm}^{-1}\right)$ relative to the adiabatic ionization energy. Bands $\mathrm{A}$ to $\mathrm{O}$ are given in Fig. 3(a). 
TABLE S9. Vibrational Structure ${ }^{a}$ of the Photoelectron Spectrum of

Trans $-\mathrm{C}_{2} \mathrm{H}_{2} \mathrm{FCl}^{+}\left(\tilde{X}^{2} A^{\prime \prime}\right)$

\begin{tabular}{|c|c|c|c|c|c|c|c|}
\hline Band & $\Delta \mathrm{E}$ & FCF & Transition & Band & $\Delta \mathrm{E}$ & FCF & Transition \\
\hline $0^{0}$ & 0 & 0.09047 & $0^{0}$ & $\mathrm{G}$ & 2814 & 0.01577 & $3^{1} 6^{1}$ \\
\hline A & 280 & 0.00359 & $9^{1}$ & $\mathbf{G}$ & 2863 & 0.02207 & $3^{1} 4^{1}$ \\
\hline $\mathbf{A}$ & 464 & 0.06213 & $8^{1}$ & & 2951 & 0.00616 & $3^{1} 8^{3}$ \\
\hline \multirow[t]{2}{*}{ B } & 928 & 0.02225 & $8^{2}$ & $\mathrm{H}$ & 2998 & 0.01285 & $3^{1} 7^{1} 8^{1}$ \\
\hline & 975 & 0.01574 & $7^{1}$ & $\mathbf{H}$ & 3118 & 0.03678 & $3^{2}$ \\
\hline $\mathbf{C}$ & 1255 & 0.02217 & $6^{1}$ & I & 3278 & 0.0126 & $3^{1} 6^{1} 8^{1}$ \\
\hline \multirow[t]{2}{*}{$\mathrm{C}$} & 1304 & 0.02340 & $4^{1}$ & I & 3327 & 0.01645 & $3^{1} 4^{1} 8^{1}$ \\
\hline & 1392 & 0.00553 & $8^{3}$ & $\mathrm{~J}$ & 3462 & 0.0056 & $3^{1} 7^{1} 8^{2}$ \\
\hline $\mathrm{D}$ & 1439 & 0.01262 & $7^{1} 8^{1}$ & $\mathbf{J}$ & 3582 & 0.02913 & $3^{2} 8^{1}$ \\
\hline \multirow[t]{4}{*}{ D } & 1559 & 0.08252 & $3^{1}$ & $\mathrm{~K}$ & 3741 & 0.00519 & $3^{1} 6^{1} 8^{2}$ \\
\hline & 1719 & 0.01624 & $6^{1} 8^{1}$ & $\mathbf{K}$ & 3791 & 0.00636 & $3^{1} 4^{1} 8^{2}$ \\
\hline & 1768 & 0.01626 & $4^{1} 8^{1}$ & & 3838 & 0.00418 & $3^{1} 4^{1} 7^{1}$ \\
\hline & 1839 & 0.0037 & $3^{1} 9^{1}$ & $\mathbf{L}$ & 4046 & 0.01191 & $3^{2} 8^{2}$ \\
\hline $\mathrm{E}$ & 1903 & 0.00519 & $7^{1} 8^{2}$ & & 4093 & 0.00705 & $3^{2} 7^{1}$ \\
\hline \multirow[t]{5}{*}{$\mathbf{E}$} & 2023 & 0.06089 & $3^{1} 8^{1}$ & M & 4373 & 0.00525 & $3^{2} 6^{1}$ \\
\hline & 2182 & 0.00618 & $6^{1} 8^{2}$ & $\mathbf{M}$ & 4422 & 0.01017 & $3^{2} 4^{1}$ \\
\hline & 2230 & 0.00406 & $6^{1} 7^{1}$ & $\mathrm{~N}$ & 4557 & 0.00639 & $3^{2} 7^{1} 8^{1}$ \\
\hline & 2232 & 0.00589 & $4^{1} 8^{2}$ & $\mathbf{N}$ & 4677 & 0.01067 & $\mathbf{3}^{3}$ \\
\hline & 2279 & 0.00423 & $4^{1} 7^{1}$ & $\mathrm{O}$ & 4836 & 0.00459 & $3^{2} 6^{1} 8^{1}$ \\
\hline \multirow[t]{3}{*}{$\mathbf{F}$} & 2487 & 0.0233 & $3^{1} 8^{2}$ & O & 4886 & 0.00813 & $3^{2} 4^{1} 8^{1}$ \\
\hline & 2534 & 0.01507 & $3^{1} 7^{1}$ & $\mathbf{P}$ & 5141 & 0.00907 & $3^{3} 8^{1}$ \\
\hline & 2559 & 0.00452 & $4^{1} 6^{1}$ & $\mathbf{Q}$ & 5604 & 0.00396 & $3^{3} 8^{2}$ \\
\hline
\end{tabular}

${ }^{a}$ Assigned according to the Franck-Condon factor computation at the

$\mathrm{B} 3 \mathrm{LYP} /$ aug-cc-pVTZ level. $\triangle \mathrm{E}$ is the excitation energy $\left(\mathrm{cm}^{-1}\right)$ relative to the adiabatic ionization energy. Bands A to $\mathrm{Q}$ are given in Fig. 4(a). 
TABLE S10. Calculated CCSD(T) Energies (in hartree) of $\mathrm{C}_{2} \mathrm{H}_{2} \mathrm{FCl}$

\begin{tabular}{lllll}
\hline & State $^{a}$ & $\mathrm{X}$ & $\mathrm{X}+$ & $\mathrm{A}+$ \\
\hline$n$ & Basis set & $1,1-\mathrm{C}_{2} \mathrm{H}_{2} \mathrm{FCl}$ & & \\
\hline 2 & aug-cc-pVDZ & -636.50113 & -636.13946 & -636.05913 \\
3 & aug-cc-pVTZ & -636.72346 & -636.35756 & -636.27732 \\
4 & aug-cc-pVQZ & -636.79027 & -636.42241 & -636.34065 \\
5 & aug-cc-pV5Z & -636.81210 & -636.44380 & -636.36121 \\
$\infty$ & CBS limit & $-636.82618(106)$ & $-636.45740(88)$ & $-636.37445(98)$ \\
\hline$n$ & Basis set & cis- ${ }_{2} \mathrm{H}_{2} \mathrm{FCl}$ & & \\
\hline 2 & aug-cc-pVDZ & -636.49794 & -636.14092 & -636.06728 \\
3 & aug-cc-pVTZ & -636.72014 & -636.35959 & -636.28556 \\
4 & aug-cc-pVQZ & -636.78701 & -636.42455 & -636.34944 \\
5 & aug-cc-pV5Z & -636.80883 & -636.44599 & -636.37038 \\
$\infty$ & CBS limit & $-636.82293(107)$ & $-636.45960(88)$ & $-636.38373(90)$ \\
\hline$n$ & Basis set & ${\text { trans }-\mathrm{C}_{2} \mathrm{H}_{2} \mathrm{FCl}}$ & & \\
\hline 2 & aug-cc-pVDZ & -636.49680 & -636.14056 & -636.06009 \\
3 & aug-cc-pVTZ & -636.71880 & -636.35916 & -636.27879 \\
4 & aug-cc-pVQZ & -636.78563 & -636.42410 & -636.34282 \\
5 & aug-cc-pV5Z & -636.80745 & -636.44555 & -636.36390 \\
$\infty$ & CBS limit & $-636.82154(107)$ & $-636.45915(87)$ & $-636.37727(85)$ \\
\hline
\end{tabular}

${ }^{a} \mathrm{X}$ stands for the neutral ground state, $\mathrm{X}+$ for the cationic ground state, $\mathrm{A}+$ for the first excited state of cation.

${ }^{b}$ Values in parentheses are errors in the last digit. 


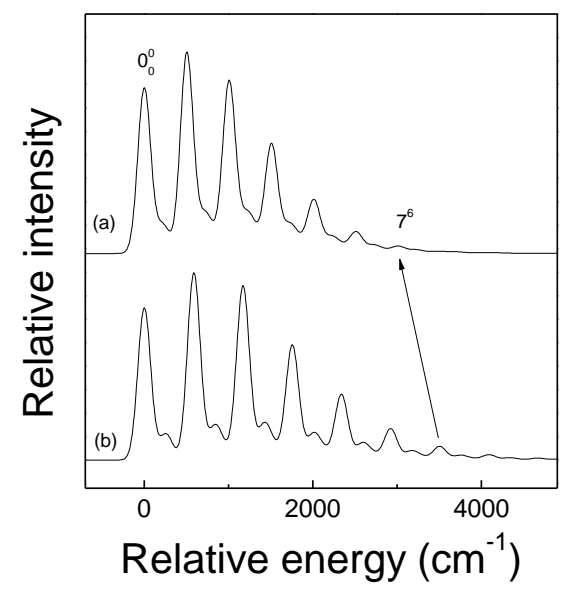

Figure S1. The TD-B3LYP simulated photoelectron spectra of $1,1-\mathrm{C}_{2} \mathrm{H}_{2} \mathrm{FCl}^{+}\left(\tilde{A}^{2} \mathrm{~A}^{\prime}\right)$ with vibrational frequencies (a) scaled by 0.86 and (b) without scaling (FWHM $=180$ $\mathrm{cm}^{-1}$.

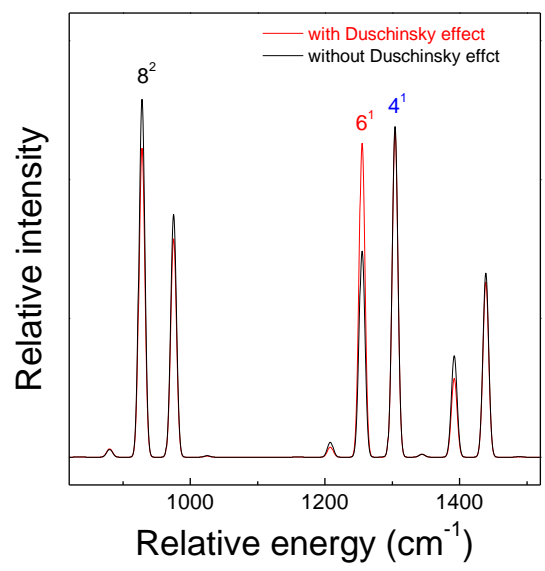

Figure S2. The B3LYP simulated photoelectron spectra of trans $-\mathrm{C}_{2} \mathrm{H}_{2} \mathrm{FCl}\left(\tilde{X}^{2} \mathrm{~A}^{\prime \prime}\right)$ with (red) and without (black) the Duschinsky effect. 


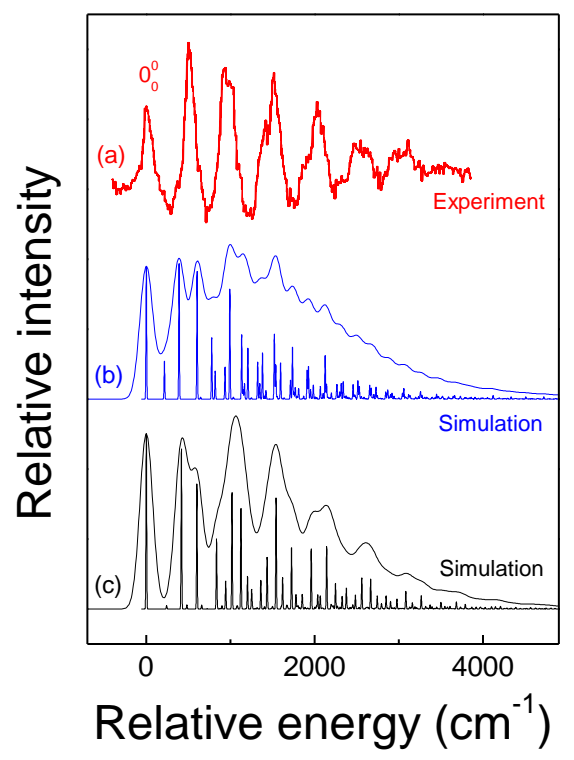

Figure S3. Photoelectron spectra of $1,1-\mathrm{C}_{2} \mathrm{H}_{2} \mathrm{FCl}^{+}\left(\tilde{A}^{2} \mathrm{~A}^{\prime}\right)$ : (a) Experimental threshold photoelectron spectrum taken from Ref. 24 (Reproduced with permission from IOP), and EOM-CCSD simulated photoelectron spectra with (b) cc-pVTZ and (c) $6-311+\mathrm{G}(\mathrm{d})$ basis sets $\left(\mathrm{FWHM}=180 \mathrm{~cm}^{-1}\right.$ and $10 \mathrm{~cm}^{-1}$ for lower and higher resolutions, respectively). The agreement between the simulated and experimental spectra is not satisfactory.

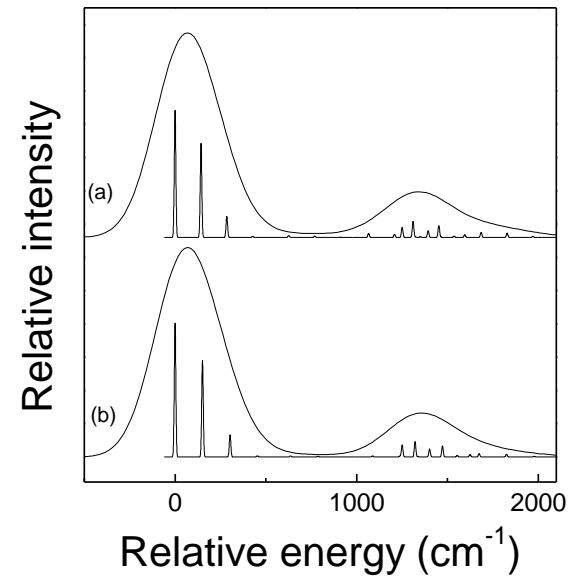

Figure S4. EOM-CCSD simulated photoelectron spectra of $c i s-\mathrm{C}_{2} \mathrm{H}_{2} \mathrm{FCl}^{+}\left(\tilde{A}^{2} \mathrm{~A}^{\prime}\right)$ with (a) cc-pVDZ and (b) 6-311+G(d) basis sets (FWHM $=350 \mathrm{~cm}^{-1}$ and $10 \mathrm{~cm}^{-1}$ for lower and higher resolutions, respectively). 


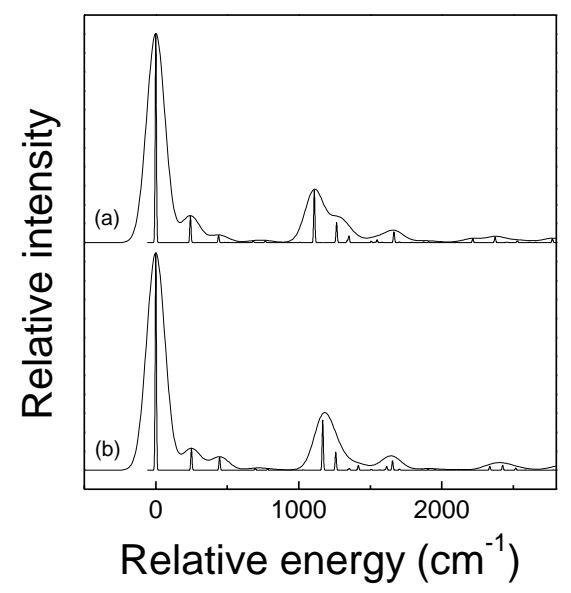

Figure S5. EOM-CCSD simulated photoelectron spectra of trans $-\mathrm{C}_{2} \mathrm{H}_{2} \mathrm{FCl}^{+}\left(\tilde{A}^{2} \mathrm{~A}^{\prime}\right)$ with (a) cc-pVDZ and (b) 6-311+G(d) basis sets (FWHM $=150 \mathrm{~cm}^{-1}$ and $10 \mathrm{~cm}^{-1}$ for lower and higher resolutions, respectively). 